is borne (Witts, 1937). Unless the patient is collapsed, when of course he should be transfused, I allow the head to be moderately raised, because the patient is more comfortable in this position than in the orthodox complete recumbency, and he can take food without swallowing troublesome quantities of air. When bleeding has ceased the ordinary indications are followed in raising the diet to the post-Lenhartz level.

\section{Results of Diet}

I will not pretend that all the patients have been able to take all the diet from the start, since some have had nausea and then they have not been pressed to eat. I do claim that they look, feel, and do much better than any previous series of cases of gastro-duodenal haemorrhage I have seen. When haemorrhage has recurred we have encouraged the patient to go on with the diet, and have tried to develop the impression that recurrence made the diet all the more vital and necessary. What have been the results? I have already described my early case where the patient died after operation. One other patient died within six hours of admission; she was known to have cirrhosis of the liver, and at necropsy she proved to have, in addition, a primary hepatic carcinoma. The remaining twenty-five did well.

The results have therefore been most satisfactory, for cases of alimentary haemorrhage which come to a general hospital include the more serious forms, and their mortality is higher than the average. The increase is not entirely explained by the disturbance of the journey, since there is a similar difference between private and public hospitals in the United States of America, where few patients are nursed in their own homes (Ledbetter, 1936). It must be attributed to the higher incidence of malnutrition and overwork among working-class patients with peptic ulcer, so that they are less able to withstand the effects of bleeding. It may be difficult to give adequate treatment such as transfusion in the home, and the risks of moving do not outweigh the benefits of hospitalization.

Seven of my patients gave a history of previous bleeding. Four of them have bled again since I treated them, one dying of cirrhosis. A few were advised to undergo surgical treatment when they had recovered from their haemorrhage. It is not my intention, however, to discuss the treatment of peptic ulcer in general, nor do I need to emphasize what is already common knowledge, that a patient who has bled once is likely to bleed again. When the patient has recovered from the haemorrhage the case must be carefully reviewed in the light, not only of the previous history and the physical examination, but also of the information provided by gastric analysis, $x$ rays, and, where possible, gastroscopy, and it must be decided whether further treatment should be medical or surgical. The patient is now fit for surgery, and the operative procedure can be suited to the pathological findings instead of being dictated by panic.

\section{Summary}

1. No reliable judgement on the relative merits of operative and non-operative treatment of gastro-duodenal haemorrhage can be based on mass hospital statistics.

2. The best individual medical results (Meulengracht, 1936) are much superior to the best individual surgical results (Finsterer, 1936).

3. It is rarely possible to differentiate between an acute and a chronic ulcer in the first forty-eight hours, which is " the optimum period for surgical attack is haematemesis" (Gordon-Taylor, 1935).
4. Treatment has been too closely centred on the bleeding point, to the detriment of the patient as a whole. Death is more often the result of haemorrhagic shock than exsanguination, and orthodox medical treatment by starvation is calculated to increase haemorrhagic shock.

5. The results of immediate feeding, supplemented when necessary by transfusion, are much superior to treatment by starvation or operation.

6. A single gastro-duodenal haemorrhage is seldom fatal. The key to the reduction of the mortality of gastro-duodenal haemorrhage lies in the prevention and treatment of recurrent bleeding. There is a legitimate field for clinical experiment in the comparison of operative and non-operative treatment of recurrent bleeding.

\section{REFERENCES}

Archer, H. E., and Graham, G. (1936). Lancet, 2, 364

Blalock, A. (1936). Bull. N.Y. Acad. Med., 12, 610.

Coller, F. A. (1936). Minnesota Med., 19, 490.

Cullinan, E. R., and Price, R. K. (1932). St. Bart's Hosp. Rep., 65,185 .

Finsterer, H. (1936). Lancet, 2, 303.

Goldman, L. (1936). J Amer. med. Assoc., 107, 1537.

Gordon-Taylor, G. (1935). Lancet, 2, 815.

Hunter, J. B. (1935). Ibid., 2, 1431.

Jennings, D. (1936). Ibid., 2, 1487.

Ledbetter, S. L. (1936). J. Amer. med. Assoc., 107, 1541

McCance, R. A. (1936). Lancet 1, 704.

Meuiengrachit, E. (1934). Acta med. scand., Suppl., 59, 375 ; (1935) Lancet, 2, 1220; (1936) Wien. klin. Wschr., 49, 1481.

Meyler, L. (1935). Acta med. scand., 87, 313.

Payne, R. T. (1936). St. Bart's Hosp. Rep., 69, 191

Schindler, R., Ortmayer, M., and Renshaw, J. F. (1937). J. Amer. med. Ass., 108, 465.

Stewart, J. D., and Rourke, G. M. (1936). J. clin Invest., 15, 697.

Witts, L. J. (1936). St. Bart's Hosp. J., 43, 167 and 182; (1937) Lancet, 1,427

Wood, I. J. (1936). British Medical Journal, 2, 115

\section{SOME OBSERVATIONS ON THE DICK TEST}

BY

FRANK L. KER, B.A.Camb., M.B., Ch.B.Ed.

Senior Assistant Medical Officer, Little Bromwich Hospital, Birmingham

In the course of routine work in a large fever hospita] where Dick testing is carried out on a considerable number of patients one is constantly meeting with negative Dick tests early in scarlet fever, with Dick-positive tests during scarlet fever convalescance, and occasionally with Dick-negative subjects developing scarlet fever. The following investigations were commenced as a result of an unusual run of such anomalous Dick reactions.

The Dick test, which was introduced as a method of assessing the susceptibility of the individual to scarlet fever, was never claimed to be as reliable as its counterpart the Schick test, although its accuracy is generally regarded to be very high. Experience in English hospitals shows that if a nurse gives a clear Dick-negative reaction she is in almost every instance safe against scarlet fever even though working in scarlet fever wards.

\section{Factors for Consideration}

There are many factors which have to be taken into consideration when testing or comparing different toxins, and the one which probably plays the greatest part in such an investigation is the accuracy with which the toxins are injected. Accuracy in performing intradermal tests is only acquired with constant practice carried out with extreme conscientiousness on the part of the operator to ensure that the whole of the $0.2 \mathrm{c.cm}$. used is injected intradermally in such a manner as to produce a good wheal. Such a wheal should be about $1 \mathrm{~cm}$. in diameter 
and be dead white, with the pores of the skin shown as small dimples on the surface. Although wheals may vary slightly in different individuals, for the purpose of comparing toxins all those in the same individual should be of the same size.

Another important factor due to the transient nature of the reaction in many cases is the conditions under which the results are read. Artificial light does not show up faint positives well, and indeed some may be classed as negative unless special care is taken if artificial light is boing employed. The best interval to allow between performing and reading the test is probably between twelve and eighteen hours. All the tests recorded below were read at three different intervals-namely, eight, twelve, and twenty-four hours after injection. At least one of these readings, and in some cases two, was done in artificial light.

The site chosen may be of importance. It is well for anyone who is contemplating doing work of this kind to test his own technique by injecting the same dose of the same toxin at two or three different sites on his own arm, or on a dozen available patients, and get a colleague to make the readings without knowing that the same toxin has been used. He will find that the majority of the readings will make "all three toxins" equal, but there will be a good deal of variation in some of the patients, and this is not referable entirely to the site chosen-assuming that all injections are done on the smooth surface of the forearm between the elbow and well above the wrist. The best comparison of two different toxins should be made at exactly corresponding sites on the two arms.

Other factors which require consideration are the strength and stability of the toxin. The more dilute the toxin the less well will it keep, and the temperature of storage has also to be taken into account, as deterioration is more marked at some temperatures than at others. In an attempt to overcome these factors various substances have been tested with a view to using them as "stabilizers."

Further questions presented themselves as regard the control fluids. Is there a pseudo-Dick reaction? How long does toxin have to be heated in order to destroy the toxin?

With all these factors to be taken into consideration it is obvious that a large series of tests has to be made to be of any value in assessing the merits of one toxin as against another. In an attempt to limit the human error to a minimum all the tests carried out were performed and read by myself. All the readings were measured in millimetres and any difference in intensity of the reactions in the same individual was carefully recorded. Any reaction less than $10 \mathrm{~mm}$. in its largest diameter was classed as an unmeasurable reaction.

Two different toxins have been used in the investigations. Toxin A diluted 1 in 1,000 was estimated to be of the same strength as Toxin B 1 in 5,000. The first series of tests to be carried out were with Toxin A to ascertain its stability to heat and the effect of storage upon it.

\section{Stability of Toxin A to Heat}

For the purpose of estimating the heat stability only known Dick-positive reactors were used, and each was tested with toxin which had been heated for one, two, or four hours respectively. Two out of twenty-two children showed a small reaction at eight hours to toxin which had been heated for one hour, while six others had at the same period very small re- actions, which could not have been mistaken for positive Dick reactions. The two that had small measurable reactions to toxin heated for one hour had small unmeasurable reactions to the toxin which had been heated for two hours. No reactions were recorded with the toxin which had been heated for four hours. The minimum time necessary to inactivate Toxin A appears, therefore, to be four hours.

\section{Stability of Toxin A on Storage}

To determine the effect of storage on Toxin A, or what may be termed the "life" of Toxin A, four samples prepared on different dates were compared. These were respectively fresh two-, four-, and six-month-old toxin which had been kept in cold storage from the date of preparation. No evidence of deterioration was found in the six- or four-month-old specimens, but slightly smaller reactions were obtained with the two-month-old sample as compared with the other three.

The reason for the apparent weakness of the two-month-old sample was difficult to explain; only later did we become alive to the bearing on the results of the composition of the stabilizing mixture used.

This series, which was carried out using only twenty individuals in each investigation, at once raised the question of the difficulties of comparing toxins as mentioned above, and the later investigations were carried out as a result on a much larger number of individuals in an effort to reduce error so far as possible.

\section{Comparison of Toxin A and Toxin B}

The second series was a comparison of Toxin A diluted 1 in 1,000 , with Toxin $B$ diluted 1 in 5,000 , and then with Toxin B diluted 1 in 3,000 . In the former comparison sixtyfive children whose average age was 4.1 years were tested. Of these fourteen were negative to all tests; twenty-two were positive to all tests, and the remaining twenty-nine showed a positive to one or other or both toxins at one or more of the readings as follows.

\begin{tabular}{|c|c|c|c|c|c|c|}
\hline \multirow{2}{*}{$\begin{array}{lll}\text { Toxin } & \ldots & \ldots\end{array}$} & \multicolumn{2}{|c|}{ At 8 hours } & \multicolumn{2}{|c|}{ At 12 hours } & \multicolumn{2}{|c|}{ At 24 hours } \\
\hline & $\mathbf{A}$ & B & $\mathbf{A}$ & B & $\mathbf{A}$ & B \\
\hline Number $+\ldots$ & 20 & 14 & 22 & 13 & 17 & 4 \\
\hline
\end{tabular}

Generally the reactions to Toxin A were a few millimetres larger than those of Toxin B in the same individual, although in a few instances the reverse was true. Toxin A produced sixteen readings which were definitely brighter than those of Toxin B in the same individual, while only four were brighter with $B$ than A. The conclusions reached were that Toxin A was the better, that the maximum number of positives were noted at the 12 -hour rcading, and that frequent readings were desirable before the result was read as negative.

When comparing Toxin A with Toxin B diluted 1 in 3,000 fifty-three children whose average age was 6.1 years were tested. Of these twenty-six were negative to all readings; fifteen were positive at all readings, and the remaining twelve showed a positive reaction at one or more readings to one or other toxin or both as follows.

\begin{tabular}{cc|c|c|c|c|c|c}
\hline & \multicolumn{2}{|c|}{ At 8 hours } & \multicolumn{2}{c|}{ At 12 hours } & \multicolumn{2}{c}{ At 24 hours } \\
\cline { 3 - 6 } Toxin $\ldots$ & $\ldots$ & A & B & A & B & A & B \\
Number + & $\ldots$ & 7 & 7 & 8 & 4 & 5 \\
\hline
\end{tabular}

The results were practically the reverse of those in the previous series. Toxin $B$ gave the slightly larger readings, and in twenty-five instances the brighter reactions. Toxin A was brighter on only two occasions. In three cases measurabie reactions occurred with both controls at the eight-hour reading comparing closely in size with the corresponding toxin reactions. Only in one subject did they persist at the twelvehour reading, and here again they were both equal in size; 
presumably this represented a true rare pseudo-reaction. None was observed at the twenty-four-hour reading. The results can be tabulated as follows.

\section{TABLE I}

$$
\begin{aligned}
& \text { Toxin A 1, 1,000 > Toxin B 1; 5,000 in } 32 \text { instances } \\
& ", \quad>\quad, \quad, \quad, \quad \text {, } \\
& \text { Toxin A } 1 / 1,000>\text { Toxin B 1/3,000 in } 3 \text { instances } \\
& \text { ". " }, \quad, \quad, 19 \text {, }
\end{aligned}
$$

The next investigation performed was to test the stability of Toxin B to heat and its "life" under varying conditions of storage.

\section{Stability of Toxin B to Heat}

In twenty-rwo cases nine positive reactions, all unmeasurabie, were noted with the toxin heated for one hour and only two with the toxin heated for two hours, which compares almost exactly with the figures obtained with Toxin $A$. The reactions, however, appeared to persist longer, three being still visible at twenty-four hours.

\section{Stability of Toxin B on Storage}

The figures obtained when using Toxin B stored for three months under varying conditions-namely, at $4^{\circ} \mathrm{C}$., at room temperature, and at $37^{\circ} \mathrm{C}$.- - showed that there was definite deterioration in all specimens as against the freshly prepared toxin, with a slight advantage for toxin kept at $37^{\circ} \mathrm{C}$. Twentyeight children were tested whose average age was 3.1 years. Of thicse, five wcre negative to all tests, five showed slight unmeasurable reactions to one or more toxins at one or more readings, nine were positive to all toxins at all readings, and the remaining nine showed a positive reaction to one toxin or more at one reading or more. At twenty-seven readings the fiesh gave definitely brighter reactions than the stored materials in the same individual, while in nearly every case the measurements were slightly greater. The smallest and/or faintest reactions were noted as follows: fifteen were with the toxin stored at $4^{\circ} \mathrm{C}$. ; fourteen with the toxin stored at room temperature, and eight with the toxin stored at $37^{\circ} \mathrm{C}$.

The results of different workers had suggested that Toxin B was somewhat less stable than $\mathrm{A}$, and further investigation was stopped in order to test the effect of adding a stabilizer. Toxin $\mathbf{B}$ was diluted 1 in 4 with stabilizer, and for the purposes of clarity this toxin will be referred to as Toxin $C$.

Specimens of Toxin $\mathrm{C}$ diluted 1 in 1,000 with boric-borate mixture stored at $4^{\circ} \mathrm{C}$. and at room temperature for six weeks vere compared with a freshly prepared toxin of the same stiength. Twenty-five children, average 3.7 years, were tested, and of these three were negative to all tests. The readings showed a very slight superiority of the freshly prepared material over the other two, but in several instances the reverse was true. It was difficult to draw any distinction between the $4^{\circ} \mathrm{C}$. and the room temperature samples, because in the majority of cases the difference was less than two millimetres and therefore quite within the limits of personal error. It would appear that Toxin $\mathrm{C}$ remains stable for a period of at least six weeks.

\section{Comparison of Toxins $\mathrm{A}, \mathrm{B}$, and $\mathrm{C}$}

Finally, Toxins A, B, and C were compared against each other on three different dates. The toxins were all prepared fresh five days before the first group of children were tested, and they were kept at room temperature until the second and third groups were tested at intervals of a week. The summary ci the results are best expressed in tabular form (see Tables II and III). The numbers in the last group are smaller than the other two on account of the fact that exactly half of the chiloren tested were negative to all three toxins, which is an unusially high figure.

In Table II the numbers in the first columns are those in which the toxins gave the largest and/or brightest reactions as compared with the other two in the same individual, while the second columns give the percentage of those numbers. It will be seen that the fresh toxins, at 60 per cent. of the readings Toxin $\mathrm{C}$ gave the best reactions, and after they had been kept at room temperature for two weeks this figure had increased to 78 per cent. Toxin $B$ had dropped from 10 per cent. to 3 per cent. during the same period.

The numbers classified as undecided refer to the readings at which at least two, and in a few cases all three, reactions in the same individual were so close, both in intensity and measurement, that no one toxin could be singled out as superior.

TABLE II.-Readings showing the Brightest and/or Largest Reactions

\begin{tabular}{c|r|r|r|r|r|r}
\hline \multirow{2}{*}{ Toxin } & \multicolumn{2}{|c|}{ Fresh } & \multicolumn{2}{c|}{ One Week } & \multicolumn{2}{c}{ Two Weeks } \\
\cline { 2 - 7 } & No. & Per cent. & No. & Per cent. & No. & Per cent. \\
\hline A & 6 & 12.5 & 12 & 17.4 & 3 & 9.4 \\
B & 5 & 10.4 & 5 & 7.2 & 1 & 3.1 \\
C & 29 & 60.4 & 45 & 65.2 & 25 & 78.1 \\
Undecided & 8 & 16.7 & 7 & 10.2 & 3 & 9.4 \\
\hline
\end{tabular}

In Table III the numbers in the first columns are those in which the toxins gave the faintest and/or smallest reactions, as compared with the other toxins in the same individual. and in the second column those numbers are expressed as percentages. In this table Toxin $\mathrm{C}$ falls from 10 per cent. of the total when the toxins are used fresh to nil when compared after two weeks, and Toxin B rises from 27 per cent. to 40 per cent. The numbers classified as undecided are those in which no toxin could be singled out as inferior to the others. Naturally this group is larger than the corresponding group in Table II. as it is much harder to distinguish between two faint tests than two bright ones.

TABLE III.-Readings showing the Faintest and/or Smallest
Reactions
\begin{tabular}{c|c|c|c|c|c|c} 
Toxin & \multicolumn{2}{|c|}{ Fresh } & \multicolumn{2}{|c|}{ One Week } & Two Weeks \\
\cline { 2 - 7 } & No. & Per cent. & No. & Per cent. & No. & Per cent. \\
\hline A & 16 & 33.3 & 22 & 31.9 & 9 & 28.2 \\
B & 13 & 27.1 & 28 & 40.6 & 13 & 40.6 \\
C & 5 & 10.4 & 4 & 5.8 & - & - \\
Undecided & 14 & 29.2 & 15 & 21.7 & 10 & 31.2 \\
\hline
\end{tabular}

The results show that over the short period there is a progressive superiority of Toxin $\mathrm{C}$ with a corresponding inferiority of Toxin B. Toxin A appears to be almost as stable as Toxin $\mathrm{C}$, but not quite so potent.

\section{Discussion}

The object of the investigation was to find:

(1) How accurately one could titrate one sample of

Dick toxin against another.

(2) How stable was ordinary Dick toxin.

(3) If certain preparations of Dick toxin were unstable, could they be stabilized?

(4) How long the toxin must be heated to destroy toxin and provide a satisfactory control fluid.

(1) It is clear from Table I that it is not an easy matter to match two toxins. It is well known that if one is trying to compare two Schick toxins on guinea-pigs one would find that if the toxins differed in strength by about 10 per cent. all the guinea-pigs would give a result in favour of the stronger toxin. Table I shows that when the comparison was made between Dick toxin A 1 in 1,000 and the weak 1 in 5,000 dilution of Toxin B, A was stronger than B, since thirty-two out of forty- 
eight patients said "greater than" and eleven said "equal"; but even here five patients out of forty-eight indicated that A was weaker than B-that is, 10 per cent. gave what is obviously an inaccurate answer. Similarly, when Toxin A was compared with Toxin B 1 in 3,000, it is clear that $\mathbf{A}$ was weaker than this dilution of $\mathbf{B}$ since nineteen patients said so, but six out of twenty-five patients gave an inaccurate answer. It is fair to conclude that if one is trying to compare two Dick toxins one must use a larger number of patients, probably about fifty, before one can get a reasonably accurate answer. This difficulty in matching two toxins and the necessity of using a large number of patients appears clearly from the careful work of Okell and Parish on over 150 patients reported by O'Brien (1930).

(2) Tables II and III indicate that Toxin B was less stable than $\mathrm{A}$ or $\mathrm{C}$. Toxin $\mathrm{B}$ was a dilution made with the ordinary boric-borate solution originally described by Glenny, Pope, and Waddington (1928) and referred to in the British Pharmacopoeia (p. 463). This diluted toxin was less stable than the toxin called $C$ in the tables, which consisted of the original toxin first diluted 1 in 4 with a stabilizing mixture, and then diluted 1 in 1.000 with the boric borate mixture. Dr. H. J. Parish of the Wellcome Physiological Research Laboratories has kindly given me this information, and told me that various stabilizing solutions are under test at the laboratories.

(3) Apparently the "stabilizer mixture" used for Toxin C has given satisfactory results, for Tables II and III indicate that the toxin has been potent up to two weeks after dilution, and later tests which we made show that six weeks after dilution this "stabilized Dick toxin" gave almost as good results as the fresh material. I understand from Dr. O'Brien that this result agrees with the laboratory investigations, and some made by other clinicians, and that his confident expectation is that this dilution is stable for much longer periods.

(4) The control Dick toxin must apparently be heated for four hours to make quite sure that the toxin is inactivated. With such a solution pseudo-reactions are extremely rare, but apparently one does encounter a patient now and then with what must be accepted as a pseudoreaction, detectable only if frequent readings are made. Pseudo-reactors are so rare that one is almost safe in dispensing with the use of the control fluid when testing ordinary patients.

\section{Summary}

Different Dick toxins can be satisfactorily matched only if the technique is carefully standardized, frequent readings are made, and a considerable number of patients - probably fifty-are available.

A properly stabilized Dick toxin dilution apparently gives when six weeks old virtually as good reactions as when freshly made up.

Dick control fluid should be heated for at least four hours.

Pseudo-reactors are very rare, but are encountered from time to time.

I am indebted to Dr. J. McGarrity, medical superintendent of the hospital, for permission to carry out these tests and for his kindly advice and encouragement; and to Dr. R. A. O'Brien of the Wellcome Physiological Research Laboratories for supplying the materials used and for his helpful suggestions.

References

O'Brien R. A. (1930). J. Hyg., 29, 357.

Glenny, A. T., Pope, C. G., and Waddington, H. (1928). J. Path. Bact., 31, 133.

\section{FURTHER OBSERVATIONS ON THE TREATMENT OF TETANUS}

\author{
BY
}

\author{
B. B. YODH, M.B., M.R.C.P., D.T.M. \& H. \\ Honorary Physician, J.J. Hospital, Bombay
}

A study of 229 cases of tetanus, with special reference to the antitoxin treatment, was recorded in a previous publication (1932). The following further observations are offered on the results of the treatment of 438 consecutive cases admitted to the J.J. Hospital from 1931 to 1935 inclusive.

Numerous papers have been published since 1932 in which the value of intrathecal injection of the antitoxin by cisternal puncture has been seriously questioned. The majority of workers advocate large doses intravenously in the early stage. Cole (1934) in a recent paper mentions that the incubation periods of our previous series were not given and that serious cases were excluded. It may be pointed out that the incubation periods of all cases were tabulated in the paper and the total mortality rate, including all serious cases, was given. The mortality rate, after deduction of cases resulting fatally within twentyfour hours of admission, was only a part of the tabulated results. The period of onset, which was not worked out in the previous series, has been included in the present one.

Recent work on the treatment of tetanus has proceeded mainly along two lines: the control of the exhausting spasms by the use of, among other things, curare and basal narcotics; secondly, the administration of a large initial intravenous dose of antitoxin. The following conclusions were reached in the previous paper:

1. That the use of tetanus antitoxin was rational and necessary.

2. The combined intrathecal (by the cisternal route), intravenous, intramuscular, and subcutaneous method gave the most promising results.

The present series of 438 cases was treated along similar lines. The antitoxin used, however, came from three different manufacturers, the potency marked on the tubes being uniform. The use of sedatives was standardized. Carbolic acid and magnesium sulphate injections were not used. Out of the total of 438 cases 209 (49.4 per cent.) were cured, 214 (50.6 per cent.) ended fatally, and 15 were discharged at request; 127 patients died within twenty-four hours of admission. Excluding these the mortality works out at 29.4 per cent. The corresponding figures for the previous series were 46.4 per cent. and 23.4 per cent. It may be mentioned that all the cases of the previous series had one brand of antitoxin

For purposes of study the cases are grouped in the following tables: I.-Age. II.-Incubation period. III.-Period of onset. IV.--Period of onset with the incubation period. V.-Type of injury.

\begin{tabular}{l|c|c|c|c|c|c}
\hline \multicolumn{8}{c}{ TABLE I. Age } \\
\hline Age .. & $1-10$ & $11-20$ & $21-30$ & $31-40$ & Above 40 & Not known \\
Number & 78 & 89 & 120 & 101 & 44 & 6 \\
Cured & $42(55.2 \%)$ & $42(49.4 \%)$ & $63(54.3 \%)$ & $45(46.4 \%)$ & $15(34.8 \%)$ & $2(40 \%)$ \\
Died & $34(44.8 \%)$ & $43(50 \%)$ & $53(45.7 \%)$ & $53(53.4 \%)$ & $28(65.2 \%)$ & $3(66 \%)$ \\
\hline
\end{tabular}

Discharged at request, 15 . 